\title{
Modeling and Simulating for Emergency Medical Service System Optimizing Based on Discrete Event System Theory
}

\author{
Tian Xie ${ }^{1}$, Yaoyao Wei ${ }^{2}$, Leilei Pan ${ }^{1}$, Tieli Wang1, Hairong Chen ${ }^{3}$ \\ ${ }^{1}$ School of Economics \& Management, University of South China, Hengyang, China \\ ${ }^{2}$ School of Political Science and Public Administration, University of South China, Hengyang, China \\ ${ }^{3}$ College of Management and Economics, Tianjin University, Tianjin, China \\ Email: "thanksky709394@163.com
}

Received 31 May 2016; accepted 18 July 2016; published 21 July 2016

Copyright (C) 2016 by authors and Scientific Research Publishing Inc.

This work is licensed under the Creative Commons Attribution International License (CC BY).

http://creativecommons.org/licenses/by/4.0/

(c) (i) Open Access

\begin{abstract}
Due to the complexity and randomness of emergency demands, it is difficult to describe and analyze the Emergency Medical Service Systems (EMSS) just by certain modeling. Therefore, a stochastic modeling and simulating method for EMSS optimizing is proposed based on the Discrete Event System theory. With a fictive and stochastic medical emergency scenario, the relevant simulation model is constructed with the FLEXSIM software quickly and explicitly. And by simulating and analyzing, it is easily to discover the bottlenecks and to optimize the EMSS system.
\end{abstract}

\section{Keywords}

Discrete Event System, Simulating, Optimizing, Emergency Medical Service System

\section{Introduction}

Various emergencies (such as natural disasters, social conflicts, and public health events) always have relevant secondary and derivatives disasters (such as group panic and casualties). Their complex evolution mechanisms cause the randomness of the disaster scenarios and the unpredictability of the emergency rescue and medical service demands. For unexpected emergency tasks, the traditional medical service systems show their weaknesses [1] [2]. Therefore, according to the emergency demands, problems desiderate to be resolved, such as how to analyze and discover the bottlenecks of the "usual EMSS system" as quickly and accurately as possible, and how to redesign a flexible "wartime EMSS system” with better layouts, service performances and service rules.

${ }^{*}$ Corresponding author.

How to cite this paper: Xie, T., Wei, Y.Y., Pan, L.L., Wang, T.L. and Chen, H.R. (2016) Modeling and Simulating for Emergency Medical Service System Optimizing Based on Discrete Event System Theory. Open Journal of Social Sciences, 4, 133-136. 
Discrete Event System (DES) is an event driven dynamic system, and its internal states change from one to another randomly. EMSS system is a typical DES system, because patient arrival rates, medical service rates, and system state change rates always show strong randomness. However, it is difficult to describe the real EMSS system and its dynamic emergency environment just by the traditional certain modeling methods, such as mathematical programming, differential equation and difference equation. Therefore, DES simulation based on distribution principles can be used as an effective tool to model, analyze, and optimize the structure and operation processes of the EMSS systems [3] [4].

As a DES simulation software based on Discrete Event System Theory (DEST), Flexsim is powerful visual modeling and analyzing functions [5]. With it, users can quickly build the 3D virtual simulation models of real EMSS systems. And by calculating and analyzing the simulation data of the model parameters, the intuitive and accurate system states can be obtained easily. And then, when we dynamically redesign the EMSS systems, the decision-making processes can be more efficient and with less cost or time.

Therefore, in this paper, a stochastic modeling and simulating method for EMSS optimizing is proposed based on DES theory. With a fictive and stochastic medical emergency scenario, the relevant simulation model is constructed with the FLEXSIM software quickly and explicitly. And by simulating and analyzing, the bottlenecks and the relevant optimal solutions are discovered.

\section{Principles for EMSS Simulating and Optimizing Based on DES}

The principle for EMSS simulating and optimizing based on DES includes steps as follows:

(1) Modeling

The abstract model of EMSS is shown in Figure 1. Based on the DES theory, the structure should be model with Flexsim software. EMSS structure consists of entities and the temporary entity flows. Entities include permanent entities which always stay in the EMSS (such as the medical service processors) and temporary entities (such as patients). And the relationships such as input, output, precondition, effect between the entities are constructed with the temporary entity flows. Secondly, the disaster scenarios and emergency demands should be analyzed and defined, and the constraints such as probability distributions of arrival interval time of patients (or patients arriving rate) should be given initially. Thirdly, the service efficiency of EMSS systems should be analyzed and defined. Some key constraints such as system capacity, types of emergency medical service, numbers of medical service processors (equipments or doctors), and service time (or service rate). Furthermore, according to the constraints, some key parameter values such as queue capacity and distances between entities should be set before simulation start.

(2) Simulating, analyzing and optimizing

After running the simulation model specified period of time, the simulation results show the steady-state statistics of key system parameters such as the waiting situations and service performances. By analyzing these data, especially the queues for medical processes, the bottlenecks of the EMSS and the relevant optimal or reconstructed solutions can be obtained.

(3) After optimizing or reconstructing, we should run, analyze and optimize the new EMSS model again and again until the simulation results satisfy the emergency medical demands. Then, the final system model can be implemented in a real scenario as satisfied solution.

\section{Example}

In this scenario, an outbreak of communicable diseases may affects about 1000 people in an area. According to this situation, the local medical facility (EMSS) sets an emergency response process including "register, diagnose,

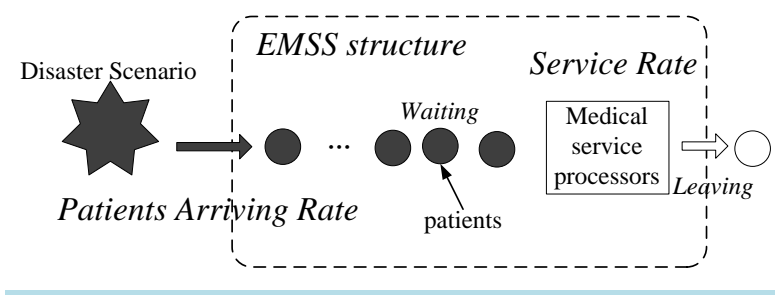

Figure 1. Abstract model of EMSS. 
and cure”. The patients are groups into levels 1 to 3 according to their affected degrees, and the EMSS has relevant three cure equipments and two diagnose equipments. Servicing performances of the equipments and some initial data of key environmental parameters include:

(1) Arrival interval time of patients obeys Exponential Distribution with mean 10 (seconds);

(2) Appearing regular of the three levels of patients obeys Discrete Uniform Distribution;

(3) Service time for registering obeys Normal Distribution with mean 5 (seconds) and standard deviation 3 (seconds);

(4) Service time for diagnosing and curing both obey Normal Distribution with mean 50 (seconds) and standard deviation 10 (seconds);

(5) All maximum capacities of each waiting queue are 200.

After about 25,000 seconds simulating, the affected 1000 people are all diagnosed and cured. As shown in the simulating process, there are 200 patients waiting for diagnosing. And there still are a large number of patients waiting for registering, which will lead the situation worse.

At the same time, the statistical data collected in the 6000 second (Figure 2) show that: firstly, most patients are in "Queue 10" waiting for diagnosing; secondly, the processing rate of diagnose "Processor 7" and "Process 9" are almost $100 \%$ (means too busy); furthermore, the average processing rate of the downstream cure service processors "Process 18 - 20" is less than $70 \%$, and there is almost no one in the "Queue 13 " waiting for cure service. It is not difficult to see that the bottleneck of this EMSS right now is in the diagnose phase. And then we should improve the performances of the diagnose processors, or added some new diagnose processors.

Therefore, two diagnose processors (processor 21 and 24) are added for improve system performance of this EMSS. And the relevant annotations of the main entity names are shown in the Table 1.

The statistical data of same time (6000 second) shows that: firstly, there are some patients waiting in "Queue 13 " for curing; secondly, the processing rates of diagnose processors and cure processors are both more than 98\%, which means that the EMSS is load balancing and effective (Figure 3); furthermore, although there still
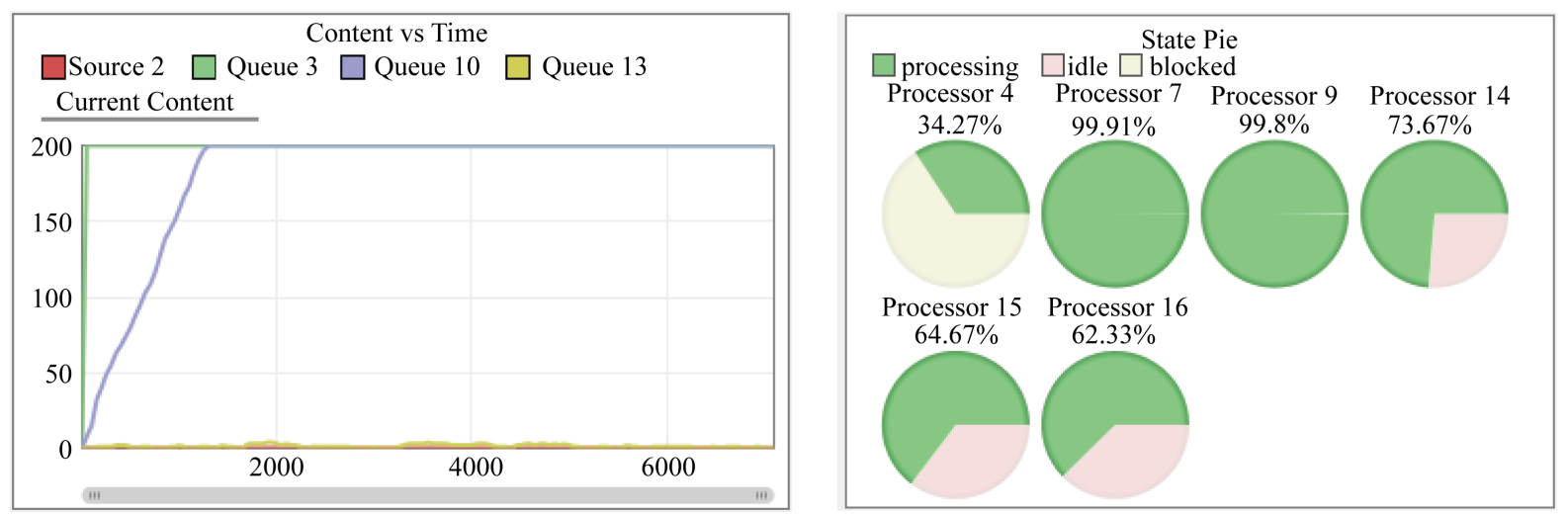

Figure 2. The screenshot of simulation data before EMSS optimizing.

Table 1 . The relevant annotations of the main entity names.

\begin{tabular}{cc}
\hline Entity Names & Relevant Annotations \\
\hline Source 2 & The source which patients come from \\
Queue 3 & Patients waiting in “Queue 3” for registering \\
Processor 4 & The processor for register service \\
Queue 10 & Patients waiting in “Queue 10” for diagnosing \\
Processor 7, 9, 21, 24 & The processor for diagnose service \\
Queue 13 & Patients waiting in “Queue 10” for curing \\
Processor 14, 15, 16 & The processor for cure service
\end{tabular}



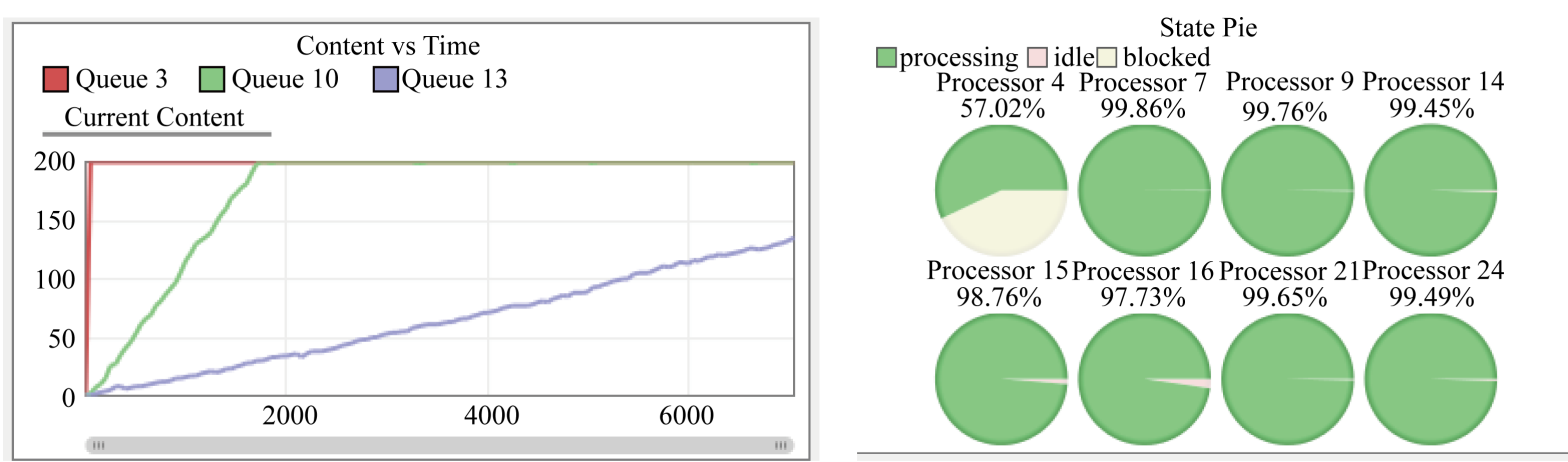

Figure 3. The screenshot of simulation data after EMSS optimizing.

are some waiting phenomenon, after 17000 seconds simulating, the all patients are cured. And with the reduced total response time, the risk of getting worse is reduced.

\section{Conclusion}

Based on DES theory, the modeling, simulating and optimizing method for EMSS is researched. And with a fictive and stochastic medical emergency scenario, the relevant simulation example is given to verify the validity of this method, and the experiment results show the advantages of it. On the one hand, DES modeling method satisfies the event-driven randomness characteristic of EMSS. Based on the object-oriented modeling, the relevant EMSS simulation model is constructed with the FLEXSIM software quickly and explicitly. On the other hand, analyzing with statistical data from simulation is easily to discover bottlenecks and to optimize the EMSS system dynamically and scientifically. And it may lead less casualties and property loss in a real disaster.

\section{Acknowledgements}

The paper is supported by Young Foundation of National Natural Science Foundation of China (No. 71501087), Young Foundation of Ministry of Education of China (No. 14YJCZH168), Young Foundation of Natural Science Foundation of Hunan Province of China (No. 2015JJ3107); Hunan Education Department Project (No. 14C0976), and Doctoral scientific research foundation of USC (No. 2013XQD27). And the research is also supported by the Key Discipline of Management Science and Engineering of USC.

\section{References}

[1] Xie, T., Li, C.D., Wei, Y.Y., Jiang, J.J. and Xie, R. (2016) Cross-Domain Integrating and Reasoning Spaces for Offsite Nuclear Emergency Response. Safety Science, 85, 99-116. http://dx.doi.org/10.1016/j.ssci.2016.01.005 http://www.sciencedirect.com/science/article/pii/S0925753516000114

[2] Baciu, F., Buglova, E., Martincic, R., Planer, R.S., Stern, W. and Winkler, G. (2010) Incident and Emergency Centre of the IAEA. Health Physics, 98, 779-783. http://dx.doi.org/10.1097/HP.0b013e3181b977e4 https://www.researchgate.net/publication/44575958_Incident_and_Emergency_Centre_of_the_IAEA

[3] Budgaga, W., Malensek, M., Pallickara, S., Harvey, N., Breidt, F.J. and Pallickara, S. (2016) Predictive Analytics Using Statistical, Learning, and Ensemble Methods to Support Real-Time Exploration of Discrete Event Simulations. Future Generation Computer Systems, 56, 360-374. http://www.sciencedirect.com/science/article/pii/S0167739X1500223X

[4] Furian, N., O’Sullivan, M., Walker, C., Vossner, S. and Neubacher, D. (2015) A Conceptual Modeling Framework for Discrete Event Simulation Using Hierarchical Control Structures. Simulation Modelling Practice and Theory, 56, 82-96. http://www.sciencedirect.com/science/article/pii/S1569190X15000647 http://dx.doi.org/10.1016/j.simpat.2015.04.004

[5] Booker, M.T, O’Connell, R.J., Desai, B. and Duddalwar, V.A. (2016) Quality Improvement with Discrete Event Simulation: A Primer for Radiologists. Journal of the American College of Radiology, 13, 417-423. http://www.sciencedirect.com/science/article/pii/S1546144015013411 http://dx.doi.org/10.1016/j.jacr.2015.11.028 